\title{
Analysis of the nonlinear dynamic response of gyroscope rotor system
}

\section{considered Elasto-Hydrodynamic Lubrication}

\author{
Cong $\mathrm{He}^{1, \mathrm{a}}$, Hongyu $\mathrm{Xu}^{1,2, \mathrm{~b}^{*}}$ and Yaoqiang Zhang ${ }^{2, \mathrm{c}}$ \\ ${ }^{1}$ School of Mechatronics Engineering, Henan University of Science and Technology, \\ Luoyang,471000, China \\ ${ }^{2}$ School of Civil Engineering, Henan University of Science and Technology, Luoyang, 471023, \\ China \\ aemail:15038688397@163.com, bemail:163xuhongyu@163.com, cemail: aq570@mail.haust.edu.cn
}

Keywords: Rolling-rotor system; EHL; Nonlinear Dynamic Response

Abstract. This essay made study of gyroscope rolling-rotor system. The composite stiffness under the state of elasto-hydrodynamic lubrication and nonlinear contact force considered the effects of minimum oil film thickness were calculated. A dynamic equation was established considered the calculated stiffness and bearing force and was numerically solved to obtain the bifurcation diagram, Poincaré maps, spectrum, phase diagrams and shaft orbit with changes of speed and radial clearance to analysis the nonlinear dynamic response of the system. The results showed that: under EHL state, with changes of speed and radial clearance, the system appears various forms of response, and there is a period-doubling bifurcation phenomenon.

\section{Introduction}

As a core component of inertial navigation system, gyroscope is widely used in satellite and other aircraft, the performance of gyroscope is largely depended on gyroscope rotor system. With the rapid development of aerospace technology, the performance of rolling bearing-rotor system for gyroscope was put forward higher requirements, bearing-rotor system dynamics research has become a hot point. Bai studied the stability and bifurcations and chaos characteristics of rolling balanced rotor system with different clearance[1]. Zhang studied bifurcations and chaos characteristics of rolling rotor system with different clearance and speed considering the influence of radial clearance on the rolling bearing stiffness[2]. Harsha[3] studied the impact of speed on nonlinear vibration of balanced rotor supported by rolling bearing and analyzed the phenomena of periodic, quasi-periodic and chaotic of rolling bearing. Cui[4] studied the chaotic behavior of the rolling bearing of flexible rotor system, the study shows that with the increase of radial clearance, the chaotic areas of the system increases. At present, the research of rolling bearings-rotor system was largely ignored the influence of oil film under EHL state. In this paper, the composite stiffness under the state of elasto-hydrodynamic lubrication and nonlinear contact force considered the effects of minimum oil film thickness were calculated, the rotor system dynamics equation was established to analysis the influence of speed and radial clearance on the response of rotor system.

\section{Dynamic Analysis Model}

In the rolling rotor system model, according to the form of whole inner bearing unit, assuming the bearing outer ring is rigidly connected with the frame, rolling balls are distributed between the inner 
and outer raceway and roll along the raceway with equal speed[5]. As shown in Fig.1.

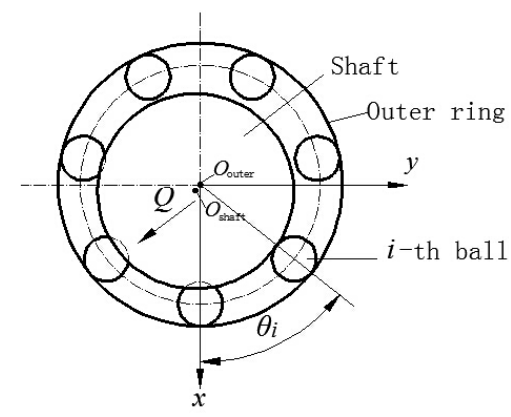

Fig.1 Rolling - Rotor System Model

Rolling Stiffness. The stiffness of rolling bearing considered the EHL has two parts, one is the contact stiffness $K_{c}$, the other is the film stiffness $K_{o}$. The overall stiffness of the rolling bearing is composite stiffness $K_{s}$ made by $K_{c}$ and $K_{o}$ in series, can be expressed as:

$$
K_{s}=\left(\frac{1}{K_{c}^{-1}+K_{o}^{-1}}\right)
$$

According to Hertz elastic contact theory, contact stiffness of two point-contact type objects can be expressed as:

$$
k=\frac{2 \sqrt{2} E}{3\left(1-\mu^{2}\right)}\left(\sum \rho\right)^{-1 / 2}\left(\delta^{*}\right)^{-3 / 2}
$$

where, $E$ is the elastic modulus, $\mathrm{N} / \mathrm{mm}^{2}, \mu$ is Poisson's ratio, $\delta *$ is the deformation coefficient, $\Sigma \rho$ is the sum of the principal curvatures of two contacting bodies.

Contact stiffness between the rolling elements and raceways can be expressed as:

$$
K_{c}=\left[\frac{1}{\left(1 / k_{o}\right)^{2 / 3}+\left(1 / k_{i}\right)^{2 / 3}}\right]^{3 / 2}
$$

where, if the material of two contacting bodies are the same, there are

$$
k_{i}=k_{o}=\frac{2 \sqrt{2} E}{3\left(1-\mu^{2}\right)}\left(\sum \rho\right)^{-1 / 2}\left(\delta^{*}\right)^{-3 / 2}
$$

According to EHL theory, film stiffness in Hertz contact area can be expressed as:

$$
K_{o}=\frac{d Q_{0}}{d h_{\min }}=6.4066 \times 10^{8} h_{\min }^{-14.6986} U^{9.31507} G^{6.7123} E^{\prime} R_{x}^{15.6986}\left(1-e^{-0.68 k}\right)^{13.6986}
$$

where, $U$ is the dimensionless velocity parameter, $G$ is the dimensionless material parameter, $E$ 'is the equivalent elastic modulus, $R_{x}$ is the curvature equivalent radius of the ball in the $x$ direction, $k$ is the ellipticity, $h_{\min }$ is the minimum film thickness between the ball and the inner and outer raceway.

$$
h_{\min }=3.63 U^{0.68} G^{0.49} W^{-0.073}\left(1-e^{-0.68 k}\right)^{13.6986}
$$

where, $W$ is a dimensionless loading parameter.

Non-linear Bearing Force. In the process of running rotor system, a layer of oil film will be produced between the rolling elements and the inner and outer raceways when considering the film thickness, and the radial bearing clearance $\lambda$ will be changed at this time. As shown in Fig. 2.

$$
\lambda=\lambda_{0}-h_{\min }=\lambda_{0}-h_{i}-h_{o}
$$


where, $\lambda_{0}$ is original radial clearance of bearing, $h_{i}$ and $h_{o}$ are respectively the minimum film thickness between rolling element and inner, outer raceway.

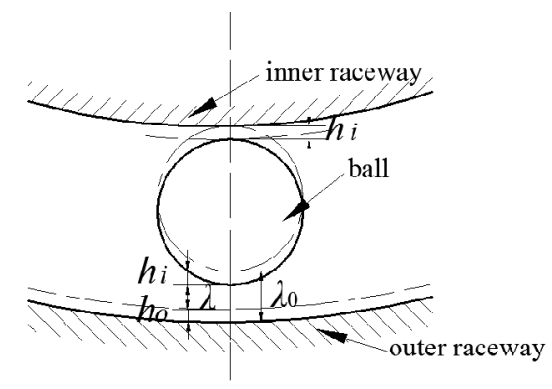

Fig. 2 The schematic of changes in radial clearance

The contact deformation $\delta_{i}$ between the $i$-th rolling element at the angular position of $\theta$ and the raceway can be expressed as:

$$
\delta_{i}=x \cos \theta_{i}+y \sin \theta_{i}-\lambda, \quad i=1,2, \cdots, N_{b}
$$

where, $x, y$ are respectively the offset of the center of rotor shaft in horizontal and vertical direction, $\lambda$ is the radial clearance, $\theta_{i}$ is the angular position of the $i$-th rolling element with respect to the $x$-axis.

$$
\theta_{i}=\frac{2 \pi}{N_{b}}(i-1)+\omega_{\text {cage }} t, i=1,2, \cdots, N_{b}
$$

where, $N_{b}$ is the number of rolling elements, $\omega_{\text {cage }}$ is the angular velocity of rolling element, $t$ is a time variable.

According to Hertz contact theory, nonlinear restoring force $F_{i}$ of the $i$-th rolling element is [6]:

$$
F_{i}=K_{s}\left(\delta_{i}\right)_{+}^{3 / 2}=K_{s}\left(x \cos \theta_{i}+y \sin \theta_{i}-\lambda\right)_{+}^{3 / 2}, i=1,2, \cdots, N_{b}
$$

subscript "+" indicates that only taking positive values in brackets, otherwise the expression in brackets take 0 .

The total resilience $F$ of bearing is:

$$
F=K_{s} \sum_{i=1}^{N_{b}}\left(x \cos \theta_{i}+y \sin \theta_{i}-\lambda\right)_{+}^{3 / 2}, i=1,2, \cdots, N_{b}
$$

The Dynamic Equation of Rolling-rotor System. According to Lagrange equation, dynamic equation of the system can be expressed as:

$$
\left\{\begin{array}{l}
m \ddot{x}+c \dot{x}+K_{s} \sum_{i=1}^{N_{b}}\left(x \cos \theta_{i}+y \sin \theta_{i}-\lambda\right)_{+}^{3 / 2} \cos \theta_{i}=m e \omega^{2} \sin (\omega t)+Q_{r} \\
m \ddot{y}+c \dot{y}+K_{s} \sum_{i=1}^{N_{b}}\left(x \cos \theta_{i}+y \sin \theta_{i}-\lambda\right)_{+}^{3 / 2} \sin \theta_{i}=m e \omega^{2} \sin (\omega t)
\end{array}\right.
$$

where, $m$ is the quality of the bearing and its parts, $\mathrm{kg}, c$ is the damping coefficient, Ns/m, $Q_{r}$ is radial force acting on the rotor, $\mathrm{N}, \omega$ is the rotor speed, $\mathrm{r} / \mathrm{min}, e$ is eccentricity of rotor (take $e=0$ ). For the influence of nonlinear factors, adaptive Runge-Kutta method was used to solve the equation of motion to analyze the nonlinear characteristics of rotor system with the variation of the parameters by bifurcation diagram, Poincaré map and FFT spectrum. 


\section{Impact of speed on the dynamic response of rotor system}

The film stiffness and minimum film thickness of rolling bearing are changed with the change of speed in the EHL state, as shown in Fig. 3 and Fig. 4, the results will impact on the dynamics of the rotor system. The displacement-speed bifurcation diagram of $x$ direction of rotor when the speed is 1000-30000r/min is shown in Fig. 5, which reflects the overall bifurcation of the dynamic response of rotor system.

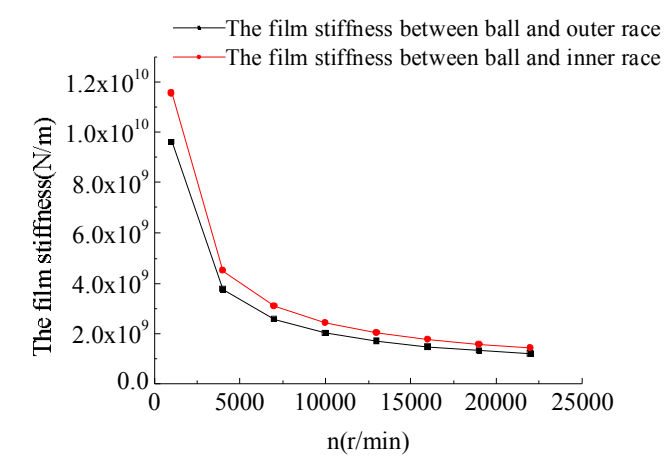

Fig.3 The film stiffness with change of speed

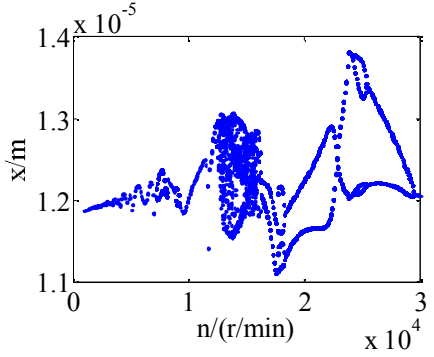

Fig.5 The displacement-speed bifurcation diagram



Fig.4 The minimum film thickness with change of speed

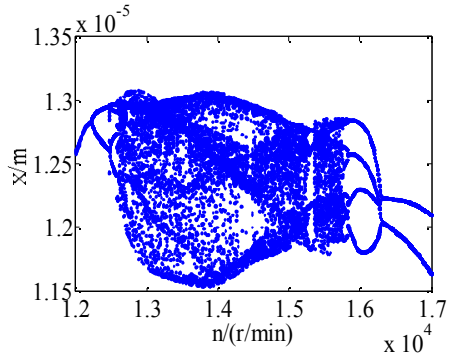

Fig.6 Enlarged bifurcation diagram

As can be seen from the bifurcation diagram, the response of the rotor system is very complicated, there are a variety of periodic and non-periodic motion. When $n<12570 \mathrm{r} / \mathrm{min}$, the system is periodic motion, which when $n=7200-8100,8900-9200,12300-12400 \mathrm{r} / \mathrm{min}$, the response of system is 2 period; when $\mathrm{n}=12490-12570 \mathrm{r} / \mathrm{min}$, it is 4 period; it is 1 period at the rest range of speed. As the speed increases, the system goes into a chaotic state. When $n>15780 \mathrm{r} / \mathrm{min}$, the system becomes periodic motion. When the system speed gradually decreases from $16400 \mathrm{r} / \mathrm{min}$ to $15700 \mathrm{r} / \mathrm{min}$, the system exists doubling bifurcation.

The Poincaré maps, spectrograms at the critical speed $(n=13500,15760,15800 \mathrm{r} / \mathrm{min})$ were studied, the results show in Fig. 7 to Fig. 9.

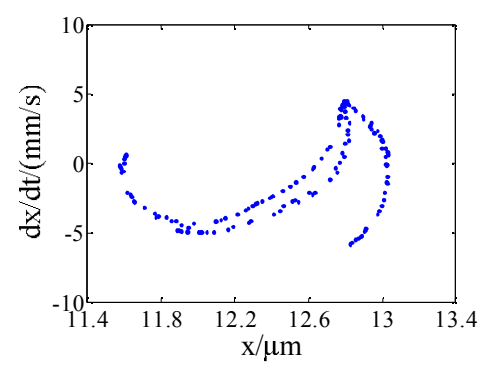

(a) Poincaré map

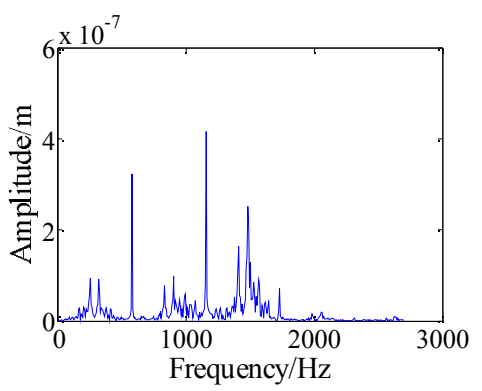

(b) Spectrogram

Fig.7 Poincaré map and spectrogram for $n=13500 \mathrm{r} / \mathrm{min}$ 


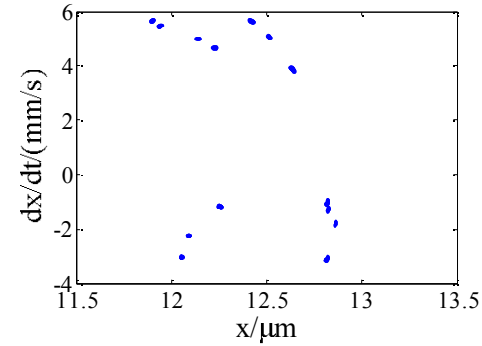

(a) Poincaré map

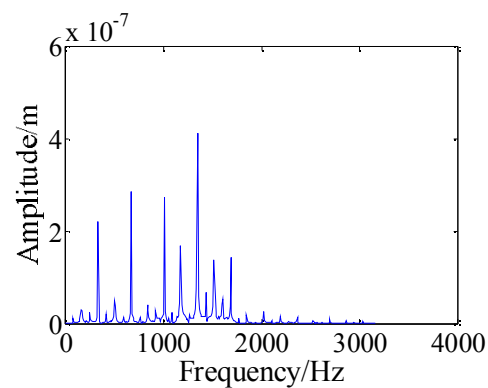

(b) Spectrogram

Fig.8 Poincaré map and spectrogram for $n=15760 \mathrm{r} / \mathrm{min}$



(a) Poincaré map

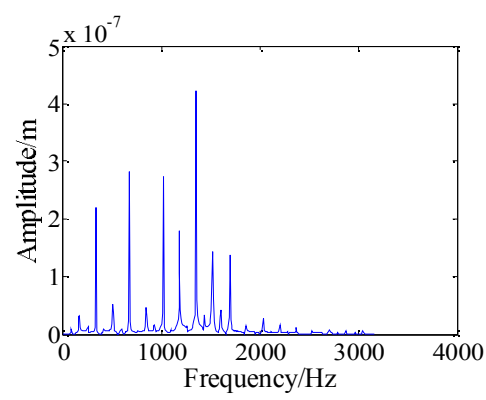

(b) Spectrogram

Fig.9 Poincaré map and spectrogram for $n=15800 \mathrm{r} / \mathrm{min}$

As can be seen, the change of speed has a significant impact on the nonlinear dynamic response of the system. Rotor system at different speeds exists various responses, such as periods, bifurcations and chaos. The system also exists doubling bifurcation phenomenon.

\section{Summary}

It is found that speed has an important influence on the nonlinear dynamic response of the rotor system by analyzing the bifurcation diagram, Poincare map and spectrum under EHL state. With the change of speed, the system has various forms of periodic and non-periodic response, and there are doubling bifurcation phenomenon. For gyroscope rotor system, a reasonable choice of operating speed will affect the stable operation of the gyroscope. Therefore, in the design stage the chaotic response speed range should be avoided to ensure the smooth running of the system.

\section{References}

[1] Liqin Zhang, Xiaolong Zhang, Yinghui Chang. The nonlinear dynamics analysis of rotor system with rolling bearings, Heavy Machinery Science and Technology. 1 (2007) 20-24.

[2] Changqing Bai, Qingyu Xu, Xiaolong Zhang. Nonlinear stability of balanced rotor due to the effect of ball bearing internal clearance, Applied Mathematics and Mechanics. 27 (2006) 159-169.

[3] S.P. Harsha, K. Sandeep, R. Prakash. The effect of speed of balanced rotor on nonlinear vibrations associated with ball bearings, Mechanical Sciences. 45 (2003) 725-740.

[4] Li Cui, Jianrong Zheng. Study on chaos behavior of flexible rotor rolling bearing system, China Mechanical Engineering. 25 (2014) 393-398

[5] Zhenzhen Guan, Haiqi Zheng, Yangang Wang. Nonlinear dynamic analysis of rolling bearing with defect localized on outer ring, Journal of Ordnance Engineering College. 21 (2009) 38-41.

[6] Harris T A. Rolling Bearing Analysis, New York: John Wiley \& Sons. (2000). 